\title{
Response of a Nonlinear String to Random Loading
}

Assistant Professor of Applied Mechanics, Division of Engineering, California Institute of Technology, Pasadena, Calif.
This paper considers the response of a nonlinear string to random excitation. It is shown that, owing to the additional stress induced by the stretching of the string, the mean squared deflection at every point is smaller than that for the equivalent linear string.

\begin{abstract}
$T_{\text {HE RESPonse of a nonlinear string to deterministic }}$ loading has been analyzed by several authors. ${ }^{1-4}$ G. F. Carrier ${ }^{1,2}$ has analyzed the free oscillations of a nonlinear string, while Lee $^{3}$ and the author ${ }^{4}$ have analyzed the response of a nonlinear string to deterministic loading. This paper is devoted to the study of a nonlinear string subjected to a random excitation. It is shown that, as in the case of deterministic loading, the additional stress induced by the stretching of the string reduces the mean squared deflection at every point compared with that for the equivalent linear string.
\end{abstract}

\section{Formulation}

Consider an elastic string, clamped at its ends subjected to a random loading. The nomenclature used is as follows:

$$
\begin{aligned}
u & =\text { transverse deflection of string } \\
T_{0} & =\text { initial tension in string } \\
\rho & =\text { linear density of string } \\
\rho \beta & =\text { viscous damping coefficient } \\
A & =\text { cross-sectional area of string } \\
E & =\text { elastic modulus of string } \\
L & =\text { length of string } \\
f(x, t) & =\text { loading on string }
\end{aligned}
$$

If the deflections and slopes are considered to be moderately small (consistent with the string remaining elastic) the equation of motion of the string is

$$
\rho\left(\frac{\partial^{2} u}{\partial t^{2}}+\beta \frac{\partial u}{\partial t}\right)=\left[T_{0}+\frac{A E}{2 L} \int_{0}^{\mathrm{L}}\left(\frac{\partial u}{\partial x}\right)^{2} d x\right] \frac{\partial^{2} u}{\partial x^{2}}
$$$$
+f(x, t)
$$

subject to the boundary conditions that

$$
u(0, t)=u(L, t)=0
$$

In the analysis that follows it will be assumed that $f(x, t)$ is a

${ }^{1}$ G. F. Carrier, "On the Nonlinear Vibration Problem of the Elastic String," Quarterly of Applied Mathematics, vol. 3, 1945, pp. 157-165.

${ }^{2}$ G. F. Carrier, "A Note on the Vibrating String," Quarterly of Applied Mathematics, vol. 7, 1949, pp. 97-101.

3 E. W. Lee, "Nonlinear Forced Vibrations of a Stretched String," British Journal of A polied Physics, vol. 8, 1957, pp. 411-413.

4 T. K. Caughev, unpublished CalTech Report No. 85, 1956.

For presentation at the West Coast Conference of the Applied Mechanics Division, Stanford, Calif., September 9-11, 1959, of TнE American Society of Mechanical Engineiers.

Discussion of this paper should be addressed to the Secretary, ASME, 29 West 39th Street, New York, N. Y., and will be accepted until one month after final publication of the paper itself in the Journal of ApPlied Mechanics.

Nore:- Statements and opinions advanced in papers are to be understood as individual expressions of their authors and not those of the Society. Manuseript received by ASME Applied Mechanics Division, November 18, 1957. Paper No. 59-APMW-4. randomly varying force with a clipped Gaussian white spectrum and has the following cross correlation:

$$
\overline{f\left(x, t_{1}\right) f\left(\eta, t_{2}\right)}=4 D \delta(x-\eta) \frac{\sin \omega_{c} \tau}{\tau}
$$

where

$$
\tau=t_{1}-t_{2}
$$

Since the eigenmodes for equation (1) are sine waves, let

$$
\begin{aligned}
& f(x, t)=\sum_{i=1}^{\infty} a_{i}(t) \sin \frac{i \pi x}{L} \\
& u(x, t)=\sum_{i=1}^{N} b_{i}(t) \sin \frac{i \pi x}{L}
\end{aligned}
$$

where $N$ is chosen such that $\omega_{N}<\omega_{c}<\omega_{N+1}$.

As a consequence of the assumed form of the cross correlation (3), the $a_{i}$ in (4) are uncorrelated and the power spectrum $g_{i}(\omega)$ of $a_{i}$ is given by

$$
\begin{aligned}
g_{i}(\omega) & =\frac{2}{L} \frac{4 D}{2 \pi} & & 0<\omega<\omega_{c} \\
& =0 & & \omega>\omega_{c}
\end{aligned}
$$

Substituting (4) into (1)

$$
\begin{aligned}
\frac{d^{2} b_{n}}{d t^{2}}+\beta \frac{d b_{n}}{d t}+ & \left(\frac{n \pi}{L}\right)^{2} \frac{T_{0}}{\rho}\left[1+\frac{A E}{2 L T_{0}} \times\right. \\
& \left.\int_{0}^{L}\left(\sum_{i=1}^{N} b_{i} \frac{i \pi}{L} \cos \frac{i \pi x}{L}\right)^{2} d x\right] b_{n}=a_{n}(t) / \rho
\end{aligned}
$$

Using the orthogonality of the circular functions, equation (6) becomes

$$
\frac{d^{2} b_{n}}{d t^{2}}+\beta \frac{d b_{n}}{d t}+\left(\frac{n \pi}{L}\right)^{2} \frac{T_{0}}{\rho}\left(1+\alpha \sum_{i=1}^{V} i^{2} b_{i}{ }^{2}\right) b_{n}=a_{n} / \rho
$$

where

$$
\alpha=\frac{A E}{4 T_{0} L}\left(\frac{\pi}{L}\right)^{2}
$$

\section{Equivalent Linear Form of Equation (7)}

The method of equivalent linearization has been applied successfully in the past to nonlinear differential equations with harmonic excitation. It will now be shown that, by a slightly different approach to the problem, this method may be applied to equation (7).

Rewriting equation (7) in the form 
$\frac{d^{2} b_{n}}{d t^{2}}+\beta \frac{d b_{n}}{d t}+\left(\frac{n \pi}{L}\right)^{2} \frac{T_{0}}{\rho} K_{n} b_{n}+\epsilon\left(b_{1}, b_{2}, \ldots b_{N}\right)=\frac{a_{n}}{\rho}$

If in (9), $\epsilon\left(b_{1}, b_{2}, \ldots b_{N}\right)$ is neglected, (9) is linear and can be solved readily. The smaller $\epsilon\left(b_{1}, b_{2}, \ldots b_{N}\right)$ is, the smaller the error in neglecting it. The logical choice of $K_{n}$ is, therefore, that value of $K_{n}$ which minimizes $\epsilon\left(b_{1}, b_{2}, \ldots b_{N}\right)$.

The choice of minimization procedure is somewhat arbitrary, but by analogy with Galerkin's method, and for mathematical expediency, it is desirable to use the minimization of the mean squared error

$$
\begin{aligned}
\overline{\epsilon^{2}}=\frac{1}{2 T} \int_{-T}^{T}\left[\left(\frac{n \pi}{L}\right)^{2} \frac{T_{0}^{\prime}}{\rho}\right]^{2} & \left\{\left[1+\alpha \sum_{i=1}^{N} i^{2} b_{i}{ }^{2}\right] b_{n}-K_{n} b_{n}\right\}^{2} d t
\end{aligned}
$$

If the process is an ergodic one, then equation (10) may be replaced by the stochastic average. Further, since the $a_{i}$ are uncorrelated

$$
\begin{aligned}
\therefore \overrightarrow{\epsilon^{2}}= & \int_{-\infty N \text {-fold }}^{\infty} \int_{-\infty}^{\infty}\left[\left(\frac{n \pi}{L}\right)^{2} \frac{T_{0}}{\rho}\right]^{2} \\
& \left\{\left[1+\alpha \sum_{i=1}^{N} i^{2} b_{i}{ }^{2}\right]-K_{n}\right\}^{2} b_{n}{ }^{2} \prod_{i=1}^{N}\left\{p\left(b_{i}\right) d b_{i}\right\}
\end{aligned}
$$

where $p\left(b_{i}\right)$ is the first probability density function of $b_{i}$.

If in $(9), \epsilon\left(b_{1}, b_{2}, \ldots b_{N}\right)$ is neglected, $b_{n}$ is Gaussian since $a_{n}$ is Gaussian.

Thus

$$
p\left(b_{i}\right)=\frac{1}{(2 \pi)^{1 / 2} \sigma_{i}} \exp \left(-b_{i}{ }^{2} / 2 \sigma_{i}{ }^{2}\right)
$$

where

$$
\sigma_{i}^{2}=\overline{b_{i}^{2}}
$$

The mean squared value of $b_{n}$ is readily computed from (17). Thus

$$
\begin{aligned}
& \sigma_{n}^{2}=\overline{b_{n}^{2}}=\int_{0}^{\infty} B_{n}(\omega) d w \\
& =\int_{0}^{\infty} \frac{2^{2}}{\rho L} \frac{D}{\pi}\left\{\left[\left(\frac{n \pi}{L}\right)^{2} \frac{T_{0}}{\rho} K_{n}-\omega^{2}\right]^{2}+[w \beta]^{2}\right\}^{-1} d w
\end{aligned}
$$

If $\beta$ is small and

$$
\begin{gathered}
\left(\frac{n \pi}{L}\right)^{2} \frac{T_{0}}{\rho} K_{n}<\omega_{c}^{2} \\
\sigma_{n}^{2} \doteq \frac{2^{2}}{2 \rho L} D\left\{\left(\frac{n \pi}{L}\right)^{2} \frac{T_{0}}{\rho} K_{n} \beta\right\}^{-1}
\end{gathered}
$$

The main contribution to the integral (18) coming from a small region close to

$$
\omega^{2}=\left(\frac{n \pi}{L}\right)^{2} \frac{T_{0}}{\rho} K_{n}
$$

By the same token it will be seen that if

$$
\left(\frac{i \pi}{L}\right)^{2} \frac{T_{0}}{\rho} K_{i}>\omega_{c}^{2}
$$

the integral (18) will be very small, thus justifying the approximate representation of $u$ in (4).

Substituting $K_{n}$ from (15) into (19)

$$
\sigma_{n}^{2}=\frac{2}{\rho L} D\left\{n^{2}\left(\frac{\pi}{L}\right)^{2} \frac{T_{0}}{\rho} \beta\right.
$$

$$
\left.\left(1+\alpha\left[\sum_{i=1}^{N} i^{2} \sigma_{i}^{2}+2 n^{2} \sigma_{n}^{2}\right]\right)\right\}^{-1}
$$

$\therefore \overrightarrow{\epsilon^{2}}=\left[\prod_{i=1}^{N}(2 \pi)^{1 / 2} \sigma_{i}\right]^{-1} \int_{-\infty N \text { fold }}^{\infty} \int_{-\infty}^{\infty}\left[\left(\frac{n \pi}{L}\right)^{2} \frac{T_{0}}{\rho}\right]^{2}\left[1+\alpha \sum_{i=1}^{N} i^{2} b_{i}{ }^{2}-K_{n}\right]^{2} b_{n}{ }^{2} \prod_{i=1}^{N}\left[\exp \left(-b_{i}{ }^{2} / 2 \sigma_{i}{ }^{2}\right) d b_{i}\right]$

Minimizing $\overline{\epsilon^{2}}$ with respect to $K_{n}$ :

$$
\begin{array}{r}
K_{n}=\left[\int_{-\infty}^{\infty} \int_{-\infty \text { fold }}^{\infty} b_{n}{ }^{2} \prod_{i=1}^{N}\left\{\exp \left(-b_{i}{ }^{2} / 2 \sigma_{i}{ }^{2}\right) d b_{i}\right\}\right]^{-1} \\
\int_{-\infty N \text { fold }}^{\infty} \int_{-\infty}^{\infty}\left[1+\alpha \sum_{i=1}^{N} i^{2} b_{i}{ }^{2}\right] b_{n}{ }^{2} \\
\prod_{i=1}^{N}\left[\exp \left(-b_{i}{ }^{2} / 2 \sigma_{i}{ }^{2}\right) d b_{i}\right]
\end{array}
$$

Evaluating the integrals:

$$
K_{n}=1+\alpha\left[\sum_{i=1}^{N} i^{2} \sigma_{i}^{2}+2 n^{2}\right] \sigma_{n}^{2}
$$

If in equation (9) $\epsilon()$ is neglected, then

$$
\frac{d^{2} b_{n}}{d t^{2}}+\beta \frac{d b_{n}}{d t}+\left(\frac{n \pi}{L}\right)^{2} \frac{T_{0}}{\rho} K_{n} b_{n}=\frac{a_{n}(t)}{\rho}
$$

The power spectrum of $b_{n}$ is therefore given by

$$
B_{n}(\omega)=g_{n}(\omega) \rho^{-1}\left\{\left[\left(\frac{n \pi}{L}\right)^{2} \frac{T_{0}}{\rho} K_{n}-\omega^{2}\right]^{2}+[\omega \beta]^{2}\right\}^{-1}
$$

where $g_{n}(\omega)$ is given by equation (5).
Noting that

$$
\frac{2}{\rho L} D\left[\left(\frac{\pi}{L}\right)^{2} \frac{T_{0}}{\rho} \beta\right]^{-1}=\sigma_{1,0^{2}}
$$

the mean squared deflection in the first mode of the linearized problem obtained by setting $\alpha=0$ in (7). Thus

$$
n^{2} \frac{\sigma_{n}^{2}}{\sigma_{1,0}^{2}}=\left\{1+\alpha \sigma_{1,0^{2}}\left[\sum_{i=1}^{N} i^{2} \frac{\sigma_{i}^{2}}{\sigma_{1,0^{2}}}+2 n^{2} \frac{\sigma_{n}^{2}}{\sigma_{1,0^{2}}}\right]\right\}^{-1}
$$

Denoting

$$
\begin{gathered}
i^{2} \frac{\sigma_{i}^{2}}{\sigma_{1,0^{2}}}=y_{i} \\
\alpha \sigma_{1,0^{2}}=z \\
\sum_{i=1}^{N} i^{2} \frac{\sigma_{i}^{2}}{\sigma_{1,0^{2}}{ }^{2}}=\sum_{i=1}^{N} y_{i}=S
\end{gathered}
$$

Equation (22) becomes

$$
2 z y_{n}{ }^{2}+[1+z S] y_{n}-1=0
$$

Solving:

$$
y_{n}=\left\{-(1+z S)+\left[(1+z S)^{2}+8 z\right]^{1 / 2}\right\}[4 z]^{-1}
$$

- See Appendix. 
The positive root being taken in (25) since $y_{n}$ by definition is positive. Multiplying both sides of (25) by $4 z$, and summing on $n$

$$
4 z S=-N(1+z S)+N\left[(1+z S)^{2}+8 z\right]^{1 / 2}
$$

Rearranging terms and squaring

$$
\left[1+z\left(1+\frac{4}{N}\right) S\right]^{2}=(1+z S)^{2}+8 z
$$

Solving for $z S$ :

$$
z S=\left\{-1+[1+4(N+2) z]^{1 / 2}\right\}\left[2\left(1+\frac{2}{N}\right)\right]^{-1}
$$

Since $z S$ is positive, the positive radical is taken.

Substituting (28) into (25), $y_{n}$ may be calculated. It will be observed from (25) that $y_{n}$ is independent of $n$, thus there is equipartition of energy among the modes.

\section{Calculation of Mean Squared Deflection $\overline{U^{2}}$}

Using equation (4)

$$
\begin{gathered}
\overline{u^{2}}=\overline{\left[\sum_{i=1}^{N} b_{i} \sin \frac{i \pi x}{L}\right]^{2}} \\
\overline{U^{2}}=\sum_{i, j}^{N} \overline{b_{i} b_{j}} \sin \frac{i \pi x}{L} \sin \frac{j x \pi}{L}
\end{gathered}
$$

Owing to the particular cross correlation function (3), the $b_{i}$ are uneorrelated: thus

$$
b_{i} b_{i}=0 \quad i \neq j
$$

Equation (30) therefore becomes

$$
\overline{U^{2}}=\sum_{i=1}^{N} \overline{b_{i}^{2}} \sin ^{2} \frac{i \pi x}{L}
$$

From equations (23) and (25)

$$
\begin{aligned}
\overline{b_{i}^{2}}=\sigma_{i}^{2} & =y_{i} \frac{\sigma_{1,0^{2}}}{i^{2}} \\
& =\frac{\sigma_{1,0^{2}}}{i^{2}}\left\{-(1+2 S)+\left[(1+2 S)^{2}+8 z\right]^{1 / 2}\right\}[4 z]^{-1}
\end{aligned}
$$

Substituting (33) into (32)

$$
\begin{aligned}
\overrightarrow{U^{2}}=\sigma_{1,0}\{ & -(1+z S) \\
+ & {\left.\left[(1+2 S)^{2}+8 z\right]^{1 / 2}\right\}[4 z]^{-1} \sum_{i=1}^{N} \frac{1}{i^{2}} \sin ^{2} \frac{i \pi x}{L} }
\end{aligned}
$$

In the linearized problem given by setting $\alpha=0$ in (7), the mean squared deflection $\overline{U_{0}} \overline{2}$ is given by

$$
\overline{U_{0}^{2}}=\sigma_{1,0^{2}} \sum_{i=1}^{N} \frac{1}{i^{2}} \sin ^{2} \frac{i \pi x}{L}
$$

Thus

$$
\frac{\overline{U^{2}}}{{U_{0}^{2}}^{2}}=\left\{-(1+2 S)+\left[(1+z S)^{2}+8 z\right]^{1 / 2}\right\}[4 z]^{-1}
$$

where $z S$ is given by equation (28).

If in $(36) z \ll 1,(36)$ reduces to

$$
\frac{\overline{U^{2}}}{\bar{U}_{0}^{2}} \doteq[1+z S]^{-1} \leq 1
$$

Further, if in (28) $N$ is large,

$$
z S \doteq 1 / 2\left[-1+(1+4 N z)^{1 / 2}\right]
$$

Using (38), equation (37) becomes

$$
\frac{\overline{U^{2}}}{\overline{U_{0}^{2}}} \doteq 2\left[1+(1+4 N z)^{1 / 2}\right]^{-1} \leq 1
$$

Thus the mean squared deflection in the nonlinear case is always less than the mean squared deflection in the equivalent linear string.

\section{Conclusions}

From the foregoing analysis it is clearly seen that the effect of the stress induced by the stretching of the string is to reduce the mean squared deflection at every point in the string compared with the corresponding mean squared deflection in the equivalent linear string.

\section{APPENDIX}

(a) Evaluation of infegral (18). Let

$$
\left(\frac{n \pi}{L}\right)^{2} \frac{T_{0}}{\rho} K_{n}=\tilde{\omega}_{n}^{2}
$$

Thus

$$
\sigma_{n}^{2}=2^{2} D / \rho L \pi \int_{0}^{\omega c}\left\{\left[\tilde{\omega}_{n}^{2}-\omega^{2}\right]^{2}+[\beta w]^{2}\right\}{ }^{-1} d w
$$

Using integral 211.16c of "Integraltaffel, Ester Teil, Unbestimmte Integral," by W. Grobner and N. Hofreiter (Springer, 1949), equation (40) becomes

$$
\begin{aligned}
& {\sigma_{n}}^{2}=2 D / \rho L \pi \tilde{\omega}_{n}\left\{\operatorname { s i n } \phi _ { 0 } \operatorname { l n } \left[\left(\tilde{\omega}_{n}^{2}+\omega_{c}^{2}+2 \tilde{\omega}_{n} \omega_{c} \cos \phi_{0}\right) /\right.\right. \\
& \left.\left(\tilde{\omega}_{n}^{2}+\omega_{c}^{2}-2 \bar{\omega}_{n} \omega_{c} \cos \phi_{0}\right)\right] \\
& \left.+\cos \phi_{0} \tan ^{-1}\left[2 \tilde{\omega}_{n} \omega_{0} \sin \phi_{0} /\left(\tilde{\omega}_{n}^{2}-\omega_{c}^{2}\right)\right]\right\} \\
& \left\{\beta^{2}\left(\tilde{\omega}_{n}^{2}-\beta^{2} / 4\right)\right\}^{-1 / 2}
\end{aligned}
$$

where

$$
\tan 2 \phi_{0}=\left\{\beta^{2}\left(\tilde{\omega}_{n}^{2}-\beta^{2} / 4\right)\right\}^{1 / 2}\left\{\tilde{\omega}_{n}^{2}-\beta^{2} / 2\right\}^{-1}
$$

If $\beta$ is small compared to $\tilde{\omega}_{n}$, equation (41) reduces to

$$
\begin{aligned}
\sigma_{n}{ }^{2} \simeq 2 D / \rho L \pi \tilde{\omega}_{n}^{2} \beta\left\{\tan ^{-1}\left[\omega_{c} \beta /\left(\tilde{\omega}_{n}^{2}-\omega_{c}^{2}\right)\right]\right. \\
\left.+0\left[\beta / 2 \tilde{\omega}_{n} \ln \left(2 \tilde{\omega}_{n} / \beta\right)\right]\right\}
\end{aligned}
$$

For $\beta / \tilde{\omega}_{n}$ very small, the second term may be neglected entirely. Thus

$$
\sigma_{n}^{2} \doteq 2 D / \rho L \pi \tilde{\omega}_{n}^{2} \beta\left\{\tan ^{-1}\left[\omega_{c} \beta /\left(\tilde{\omega}_{n}^{2}-\omega_{c}^{2}\right)\right]\right\}
$$

If $\omega_{c}>\tilde{\omega}_{n}$

$$
\sigma_{n}^{2} \doteq 2 D / \rho L \tilde{\omega}_{n}^{2} \beta
$$

If $\tilde{\omega}_{n}>\omega_{c}$

$$
\begin{aligned}
\sigma_{n}^{2} \pm 2 D / \rho L \pi \tilde{\omega}_{n}^{2} \beta\left\{\omega_{c} \beta /\left(\tilde{\omega}_{n}^{2}-\omega_{c}^{2}\right)\right\} \\
=2 \beta D \omega_{c} / \rho L \pi \beta \tilde{\omega}_{n}^{2}\left(\tilde{\omega}_{n}^{2}-\omega_{c}^{2}\right) \\
\ll 2 D / \rho L \pi \beta \tilde{\omega}_{n}^{2} \text { for } \beta / \tilde{\omega}_{n} \ll 1
\end{aligned}
$$

This justifies the approximate representation of $u$ in equation (4)

(b) Justification for neglecting term $z \sum_{N+1}^{\infty} i^{2}\left(\sigma_{n}{ }^{2} / \sigma_{1,0}{ }^{2}\right)$. As is shown in (46), for $\tilde{\omega}_{i}^{2}>\omega_{c}^{2}$

$$
\sigma_{i}^{2} \doteq 2 D \omega_{c}\left[\rho L \pi \tilde{\omega}_{i}^{2}\left(\tilde{\omega}_{i}^{2}-\omega_{c}^{2}\right)\right]^{-1}
$$


i.e.,

$$
\begin{gathered}
i^{2} \frac{\sigma_{i}{ }^{2}}{\sigma_{1, \theta^{2}}} \doteq \omega_{c} \beta\left[\pi\left(\omega_{i}^{2} K_{i}-\omega_{e}{ }^{2}\right)\right]^{-1} \\
\therefore \sum_{N+1}^{\infty} i^{2} \frac{\sigma_{i}^{2}}{\sigma_{1,0^{2}}} \simeq \frac{\omega_{c} \beta}{\pi \omega_{0}{ }^{2} K_{N+1}} \sum_{N+1}^{\infty}\left(i^{2}-\frac{\omega_{c}{ }^{2}}{\omega_{0}{ }^{2} K_{N+1}}\right)^{-1} \\
\leq \frac{\beta}{2 \pi \omega_{0} K_{N+1}{ }^{1 / 2}} \int_{N+1}^{\infty}\left[\left(i-\frac{\omega_{c}}{\omega_{0} K_{N+1}{ }^{1 / 2}}\right)^{-1}\right. \\
\left.-\left(i+\frac{\omega_{c}}{\omega_{0} K_{N+1}{ }^{1 / 2}}\right)^{-1}\right] d i \\
\leq \frac{\beta}{2 \pi \omega_{0} K_{N+1}{ }^{1 / 2}} \ln \left[\left(N+1+\omega_{c} / \omega_{0} K_{N+1}{ }^{1 / 2}\right) /\right. \\
\left.\left(N+1-\omega_{c} / \omega_{0} K_{N+1}{ }^{1 / 2}\right)\right] \\
\doteq \frac{\beta}{2 \pi \omega_{0} K_{N+1}{ }^{1 / 2}} \ln [(N+2) / N]
\end{gathered}
$$

\section{Comparing}

$$
\begin{gathered}
\sum_{N+1}^{\infty} i^{2} \frac{\sigma_{i}^{2}}{\sigma_{1,0^{2}}} \text { with } \sum_{i=1}^{N} i^{2} \frac{\sigma_{i}^{2}}{\sigma_{1,0}{ }^{2}} \\
\sum_{N+1}^{\infty} i^{2} \frac{\sigma_{i}^{2}}{\sigma_{1,0^{2}}} / \sum_{1}^{N} i^{2} \frac{\sigma_{i}{ }^{2}}{\sigma_{1,0^{2}}} \\
\doteq \frac{2 \beta\left(1+\frac{2}{N}\right) z \ln [(N+2) / N]}{2 \pi \omega_{0} K_{N+1}{ }^{1 / 2}\left\{-1+[1+4(N+2) z]^{1 / 2}\right.}
\end{gathered}
$$

For $z$ small

$$
\sum_{N+1}^{\infty} i^{2} \frac{\sigma_{i}^{2}}{\sigma_{1,0^{2}}} / \sum_{1}^{N} i^{2} \frac{\sigma_{i^{2}}}{\sigma_{1,0^{2}}} \leq \frac{\beta z^{1 / 2}}{\omega_{0}} 0\left[\frac{\ln (1+2 / N)}{N}\right]
$$

If $\beta$ is small and $N$ is large, this ratio becomes very small and may be neglected, thus justifying the treatment given in the paper. 